\title{
Karyological Studies on Black Rat (Rattus rattus L.) from Bulgaria
}

\author{
Rossitsa Belcheva and Vulko Bisserkov \\ Chair of Genetics, Faculty of Biology, University \\ of Sofia, Sofia, Bulgaria
}

Received October 15, 1982

Karyological investigations on the black rat (Rattus rattus) have shown that it is a unique species because of frequent occurrences of chromosome polymorphism and geographical variations due to Robertsonian fusions, pericentric inversions and supernumerary chromosomes. The black rats $(R$. rattus) are divided into the following four geographical variants according to the difference of chromosome number and karyotypes: the Asian $(2 n=42)$, Ceylones $(2 n=40)$, Oceanian $(2 n=38)$, and Mauritius ( $2 \mathrm{n}=42)$ (Yosida et al. 1971, 1974, Yosida 1979a, b). They distribute occupying different geographical areas (Yosida et al. 1980). It is suggested that the Asian karyotype is the ancestral form and that the Ceylonese and the Oceanian karyotypes developed sequentially by centric fusions (Yosida et al. 1971, 1974, Yosida 1973). The Mauritius karyotype developed from the Oceanain by the Robertsonian fission type mechanism (Yosida et al. 1979). All R. rattus subspecies distributed outside of Asia have a diploid chromosome number of 38 (Gamperl 1980).

European black rats have been studied karyologically by many investigators (Capana et al. 1970, 1971, Kral 1974, Pretel et al. 1978, Vistorin et al. 1978, Dulic 1978, De La Guardia et al. 1979, Gamperl 1980). Polymorphism caused by supernumerary chromosomes was only described for a Spanian population of European black rat (Pretel et al. 1978). De La Guardia et al. (1979) have found a structural polymorphism caused by changes in the morphology of the chromosomes in three pairs.

The present paper reports the karyological studies of $R$. rattus collected from different areas of Bulgaria, which reveal a structural rearrangement of some autosomes, B-chromosomes, C-band polymorphism and the location of active ribosomal cistrons.

\section{Materials and methods}

The specimens of $R$. rattus ( 13 male and 12 female) used for this study were collected from 7 localities in Bulgaria (Fig. 1). Chromosome preparations were made from colchicine-blocked metaphases of bone marrow cells of the femur. Preparations were also made from the testes of all the male animals to give both mitotic and meiotic metaphases. The technique was that of Evans et al. (1964). G-banding was accomplished with the method of Seabright (1971), using Giemsa instead of Leischmann's stain and C-banding was done by the method of Sumner 
(1972). For selective staining of the active nucleolar organizing regions (NORs) the technique of Goodpasture and Bloom (1975) was applied.

\section{Results and discussion}

The karyotypes in 25 black rats, $R$. rattus, from 7 localities in Bulgaria have been analyzed. Among them 14 showed the typical Oceanian type with 38 chromosomes and among the remaining 11 rats from 4 localisties 8 were characterized by having 39 chromosomes and 3 rats had 40 chromosomes. They included one and two supernumerary chromosomes respectively (the B-chromosomes are described in the end of this paper). The Oceanian karyotype is characterized by two large metacentric pairs $\left(M_{1}\right.$ and $\left.M_{2}\right)$. According to Yosida et al. $(1971,1974)$ the larger metacentric pair $\left(M_{1}\right)$ has originated in the Robertsonian fusion of acrocentric pairs no. 4 and 7 , and the other smaller one $\left(M_{2}\right)$ in that of the acrocentric pairs no. 11 and 12 in the Asian type of black rat. There are six small metacentric pairs (nos.

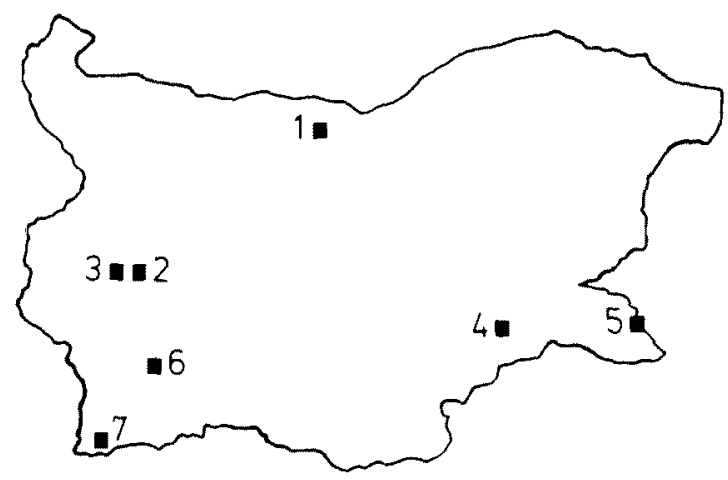

Fig. 1. A map of Bulgaria showing the localities from where rats were trapped.

$14,15,16,17,19$ and 20), two subtelocentric chromosome pairs (nos. 1 and 9), seven acrocentric pairs (nos. 2, 3, 5, 6, 8, 10 and 13) in the Oceanian karyotype, and acrocentric $X$ and $Y$.

In the karyotypes analysed we found a polymorphic pair no. 13 in respect to acro- and subtelocentrics. This polymorphism of chromosome no. 13 is due to a pericentric inversion. Among the 25 specimens of $R$. rattus karyologically studied 17 have an acrocentric pair no. 13 (A/A), 2 have a subtelocentric pair no. 13 (S/S), and 2 have a heteromorphic pair no. 13 (A/S) with one member acrocentric and one member subtelocentric (Figs. 2, 3, 4). Four individuals from 4 localities (1, 2, 3, 4-Fig. 1) show a mosaicism in respect to the shape of chromosome no. 13. Some metaphases have an acrocentric pair no. 13 (A/A), and some metaphases have a heteromorphic pair no. 13 (A/S). Yosida (1977a) described a wide distribution of pair no. 13 chromosome polymorphism in the rats and suggested that the inversion of the pair has occurred independently in several places of the world. Our findings are in agreement with this suggestion.

In four individuals from 3 localities (2,4 and 7-Fig. 1) we found a polymor- 
phism in chromosome pair no. 10 due to a pericentric inversion. These four individuals show a mosaicism in respect to the type of chromosome no. 10. Some metaphases have an acrocentric chromosome pair no. 10 (A/A), and some metaphases have a heteromorphic pair no. 10 with one acrocentric and one subtelocentric chromosome (A/S) (Fig. 5).

In two individuals from two localities (5 and 7-Fig. 1) a pericentric inversion in chromosome pair no. 9 was established. These two individuals show a mosa-

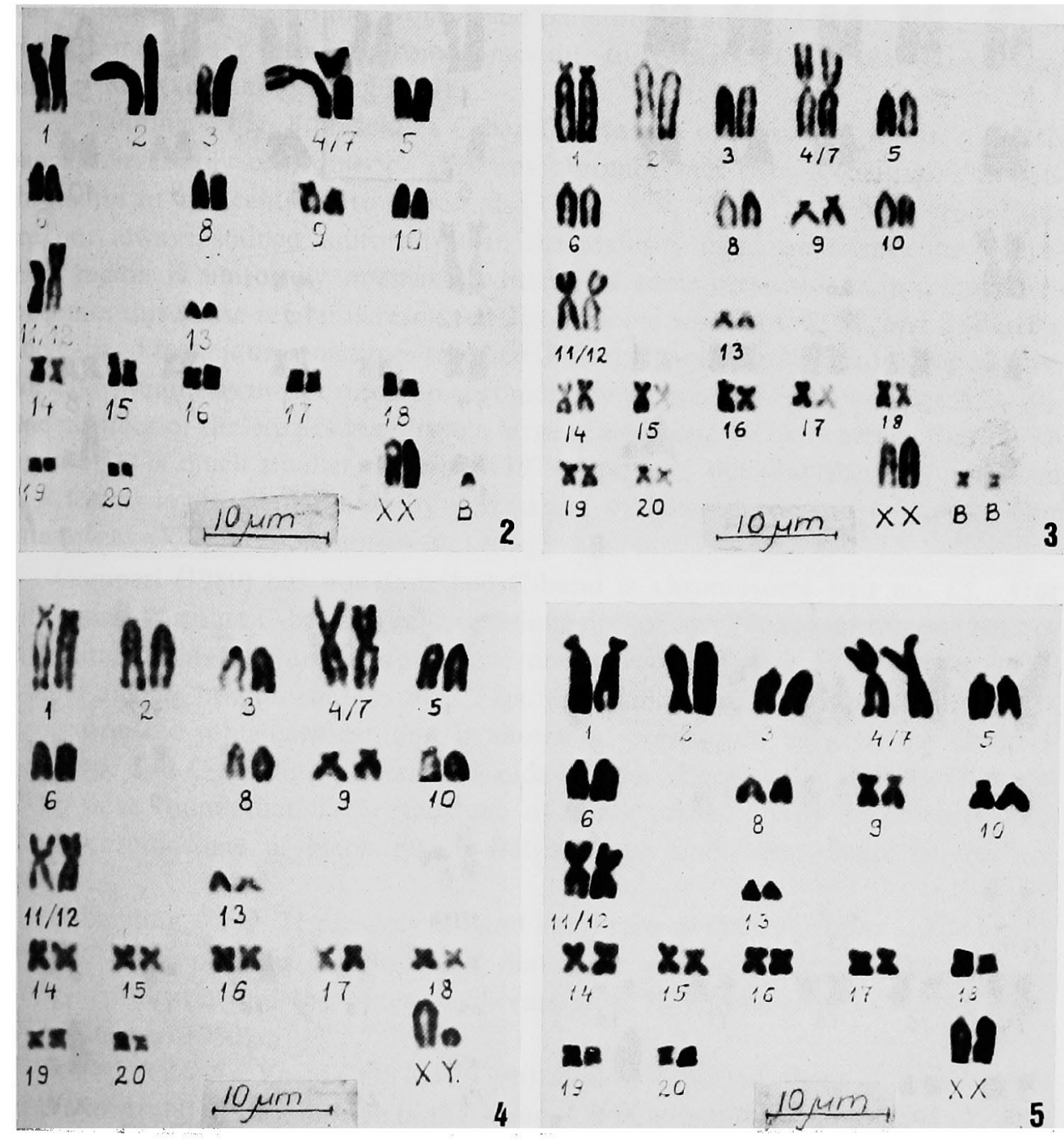

Figs. 2-5. 2, karyotype of female $R$. rattus with acrocentric pair no. 13, with Robertsonian fission in one member of $\mathrm{M}_{1}$ resulted in the formation of two acrocentric chromosomes, with a heterozygous $\mathrm{M}_{2}$ and one acrocentric B-chromosome. 3, karyotype of female $R$. rattus with subtelocentric pair 13 and with two metacentric B-chromosomes $(2 \mathrm{n}=38+2 \mathrm{Bs}) .4$, karyotype of a male $R$. rattus with heteromorphic pair no. 13 consisting of acrocentric and subtelocentric chromosomes. 5 , karyotype of female $R$. rattus with heteromorphic pair no. 10 consisting of acrocentric and subtelocentric chromosomes, and heteromorphic pair no. 9 consisting of subtelocentric and metacentric chromosomes. 
icism in respect to the type of the chromosome no. 9. Some metaphases have a subtelocentric chromosome pair no. 9 (S/S), and some metaphases have a heteromorphic chromosome pair no. 9 consisting of one subtelocentric and one metacentric chromosome (S/M) (Fig. 6). Polymorphism in pairs no. 1, 9 and 13 chromosomes have been described in black rats (Yosida 1977a). The pair no. 1 and 9

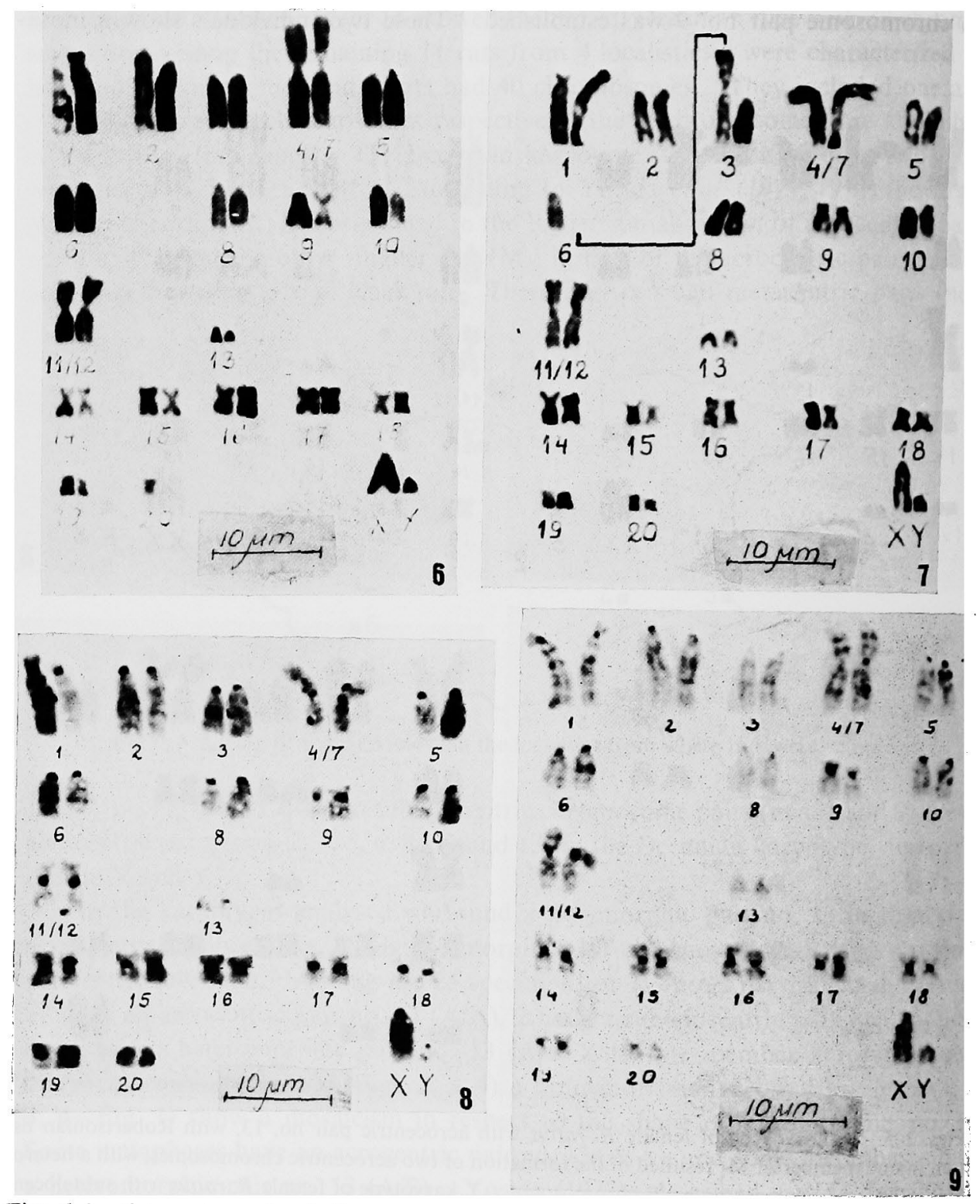

Figs. 6-9. 6, karyotype of male $R$. rattus with heteromorphic pair no. 9 consisting of subtelocentirc and metacentric chromosomes. 7, karyotype of male $R$. rattus with Robertsonian fusion in chromosome pairs nos. 3 and 6 . 8, C-band karyotype of male $R$. rattus. 9, C-band karyotype of male $R$. rattus. Note the samll amount of $\mathrm{C}$-band material on chromosome pairs 3 and 8 and the absence of C-band on chromosome pair 13. 
polymorphism was found only in Asian type black rats. Recently new cases of structural polymorphism were discovered in this species caused by pericentric inversions affecting new chromosome pairs nos. 5 and 7 (according to Yosida's numeration) (De La Guardia et al. 1979), and pair no. 8 (Kasahara et al. 1981). Our data indicate two new inversions in chromosomes nos. 9 and 10 in black rat. These inversions were not established in all metaphases analysed per individual and we still refrain for commenting their importance.

In one individual we found metaphases with 37 chromosomes because there was Robertsonian fusion in chromosome pairs nos. 3 and 6 (Fig. 7). In one individual a metaphase with 39 chromosomes due to Robertsonian fission-type mechanism in $\mathbf{M}_{1}$ was established (Fig. 2).

C-banding. Fig. 8 presents a C-band karyotype of a male $R$. rattus. The Cband is invariably centromeric. The sex chromosomes carry constitutive heterochromatin at the centromere region also (Figs. 8, 9, 10). The centromere regions are not always stained uniformly. In the majority of chromosomes the centromere region is uniformly intensive, whereas in some preparations in a few chromosomes this dense region is resolved, chromosome pairs nos. 3, $M_{1}$ and 8 (Fig. 9). The C-band technique sometimes revealed dimorphism in certain chromosome pairs. We have found this most evident in chromosome pairs nos. 14 and 16 (Figs. 8, 9, 10). One member of these pairs may have a large centromeric block, whereas that on its homologue is much smaller. Yosida (1979a) pointed out that the polymorphism in $R$. rattus is also remarkable by a variation of heterochromatic C-bands. Our data reveal a $C$-band polymorphism too.

Gamperl (1980) has not described C-band in chromosome pair no. 13. Our data showed distinct C-band in centromeric region of pair 13, no centromeric heterochromatin in this pair and dimorphism: one member of pair 13 has centromeric C-band and its homologue has not (Figs. 8, 9, 10). Pair 13 is polymorphic for acrocentric and subtelocentric, and it shows no correlation between the chromosome type and C-banding pattern. Yosida (1975), Vistorin et al. (1978), Gamperl (1980) have found that Y-chromosome of black rat is totally heterochromatic. The Y-chromosome of black rat in Bulgaria has only centromeric heterochromatin.

G-banding. Fig. 11 presents G-band karyotype of male $R$. rattus. The analyses of G-band patterns demonstrated that there are no differences between $R$. rattus in Bulgaria and the other black rats with 38 chromosomes (Yosida et al. 1973, Gamperl 1980).

Ag-NORs. As Yosida $(1978,1979 b)$ already reported there is a consistent pattern of Ag-NOR distribution in the Asian, Ceylones and Oceanian types of black rats $R$. rattus. This species has $3 \mathrm{Ag}$-NORs bearing chromosome pairs nos. 3, 8 and 13. In our material, chromosme analysis after application of silver staining technique (Goodpasture and Bloom 1975) indicated that there are four chromosome pairs with silver stained NORs. Silver staining occurred at the telomere region of the short arms of chromosome pairs 3,8 and 13 , and at the telomere region of the long arm of one acrocentric chromosome which is identified as chromosome 10 (Fig. 12). The NORs in chromosomes 3,8 and 13 are localized close but distally to the 


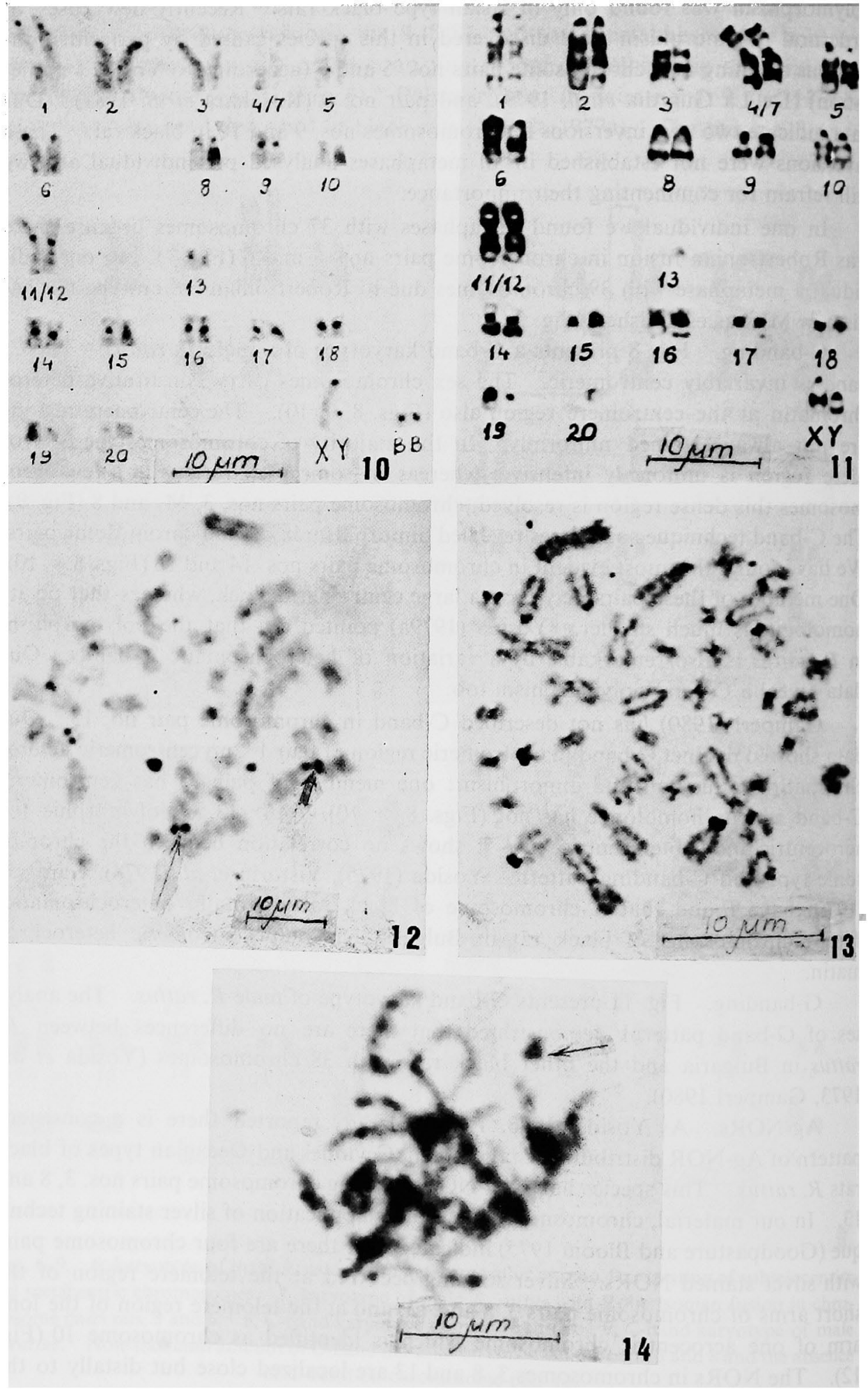


centromeric heterochromatin. Chromosomes of $R$. rattus show silver stained NORs in one or both of the homologues. The number of chromosomes per cell on which Ag-NORs could be detected vary (Fig. 13). NOR association is observed among chromosomes nos. 3 and 8 (Fig. 12).

The ammoniacal silver staining procedure which stains the chromosome regions containing the rRNA genes indicates only active NOR (Hsu et al. 1975, Miller et al. 1976). It seems likely that silver staining involves non-histon proteins (Howell and Denton 1976) which are acidic proteins involved in rRNA transcription (Olert et al. 1979) as a part of the ribosomal nucleoprotein complex remaining around the active NORs (Schwarzacher et al. 1978). With respect to the total number of NORs in Rattus rattus genome one has to await the results of in situ hybridization with radioactively labeled rRNA.

Supernumerary, or B-chromosomes. As was already pointed out there are 11 specimens of black rats from 4 localities (1, 2, 3 and 7-Fig. 1) with a presence of 1 or 2 supernumerary chromosomes, respectively. The size and the shape of these chromosomes are different. In 6 specimens supernumeraries are metacentric. These additional metacentric chromosomes in size belong to the category of the 20th pair of metacentric (Figs. 3, 10). Four individuals have one metacentric B-chromosome, and two have 2 metacentric B-chromosomes. In 5 specimens from two localities (3 and 7-Fig. 1) there are acrocentric B-chromosomes (Fig. 2). Four individuals have one acrocentric B-chromosome and the remaining one has 2 acrocentric B-chromosomes.

In male meiotic prophase-I B-chromosomes appear in a univalent condition. At pachytene it is possible to observe that the B-chromosome is strongly contracted and it seems to a metaphase chromosome (Fig. 14). The observations made in meiosis clearly show the supernumerary character of these chromosomes.

In the Oceanian-type black rat, supernumerary chromosomes have been reported for the black rats in Southern India (Yosida 1977b) and for European black rats from a Spanian population (Pretel et al. 1978). The superunmerary chromosomes in the black rats are metacentric only (Yodida 1977b, Pretel and De La Guardian 1978). In the individuals with extra chromosomes each of the additional metacentic or acrocentric supernumerary chromosomes show a clear centromeric heterochromatin (C-band) only (Fig. 10). Raman and Sharma(1974) reported no centromeric $\mathrm{C}$-band in the B-chromosomes of black rats. Yosida found that "... The supernumeraries ... stained heavily along their entire length by C-band staining" (Yosida 1977b, p. 149). Our findings about C-band in B-chromosome in $R$. rattus are not in agreement with Yosida's data. Evidently there are different ways in which B-chromosomes in black rats might have arisen, and they might have differed in size, shape, C-bands and in function as well.

Figs. 10-14. 10, C-band karyotype of male R. rattus $(2 \mathrm{n}=38 / 2 \mathrm{Bs})$. Note the small amonut of Cband material on $\mathrm{M}_{1}$ and clear $\mathrm{C}$-band in centromeric regions on the B-chromosomes and $\mathrm{Y}$. 11 , G-band karyotype of male $R$. rattus. 12 , silver-stained metaphase plate of male $R$. rattus. Note NOR in telomeric region of the long arm of chromosome 10 (long arrow) and association between NORs of chromosomes 3 and 8 (short arrow). 13, silver-stained metaphase plate of male $R$. rattus. Note Ag-NORs of different size. 14, pachytene stage from male meiosis of $R$. rattus. Arrow indicates B-chromosome. 


\section{Summary}

The black rat (Rattus rattus L.) from Bulgaria belongs to the Oceanian-type $(\mathrm{n}=38)$. Chromosomal analyses show that a polymorphism occurs in chromosomes nos. 9,10 and 13 due to pericentric inversions. A polymorphism concerning size difference of centromeric heterochromatin (C-band) in some of the chromosomes is observed. The analyses of G-band patterns demonstrate that there are no differences between our material and the other black rats with 38 chromosomes. The NORs of the black rat $(R$. rattus $)$ are found in telomeric regions of the short arms of chromosome pairs $3,8,13$ and in telomeric region of the long arm of chromosome pair no. 10. A polymorphism due to supernumerary chromosomes is detected. The supernumeraries are metacentric and acrocentric. They show centromeric $\mathrm{C}$-band after $\mathrm{C}$-band staining.

\section{References}

Capanna, E., Civitelli, M. V. and Nezer, R. 1970. The karyotype of the black rat (Rattus rattus L.). Another population with a 38-chromosome complement. Experentia 26: 422-425.

-, - and - 1971. On the chromosome polymorphism of Rattus rattus L: A study on WestEuropean populations. Experentia 27: 583-584.

De La Guardia, R. D., Ladron de Guevara, G. R. G. and Pretel, M. 1979. Chromosomal polymorphism, caused by pericentric inversions in Rattus rattus ssp. frigivorus (Raf.) from the South-East of the Iberian Peninsula. Genetica 51: 103-106.

Dulic, B. 1978. Chromosomes of small mammals from the southwestern karstic regions of Yugoslavia. The II Congr. Theriol. Internat., Brno.

Evans, E. P., Breckon, G. and Ford, C. E. 1963, An air-drying method for meiotic preparations from mammalian testes. Cytogenetics 3: 289-194.

Gamperl, R. 1980. Karyological studies on European rats. Genetica 52/53; 87-92.

Goodpasture, C. and Bloom, S. E. 1975 . Visualization of nucleolar organizer regions in mammalian chromosomes using silver staining. Chromosoma (Berl.) 53: 37-50.

Howell, W. M. and Denton, I, E. 1976. Negative silver staining in A-I and satellite DNA-rich regions of human chromosomes. Chromosoma, (Berl.) 57: 165-169.

Hsu, T. C., Spirito, S. W. and Pardue, M. L. 1975. Distribution of $18+28$ S ribosomal genes in mammalian genomes. Chromosoma (Berl.) 53: 25-36.

Kasahara, S. and Yonenaga-Yasuda, T. 1981. Chromosome variability in Brazilian specimens of Rattus rattus $(2 \mathrm{n}=38)$. Experientia 37: 31-32.

Kral, B. 1974. Formation of karyotype of rats of the subgenus Rattus. Sympos. Theriol. II Porc. Intern. Sympos. Species Zoogeogr. Europ. Mammals, Prage: 237-245.

Miller, D. A., Dev, V. G., Tantravahi, R. and Miller, O. J. 1976. Supprssion of human nucleolus organizer activity in mouse-human somatic hybrid cells. Expl. Cell Res. 101: 235245.

Olert, J., Sawatzki, G., Kling, H. and Gebaner, J. 1979. Cytological and histochemical studies on the mechanism of the selective silver staining of nucleolus organizor regions (NORs). Histochemistry $60: 91-99$.

Pretel, M. A. and De La Guardian, D. G. R. 1978. Chromosomal polymorphism caused by supernumerary chromosomes in Rattus rattus ssp. frigivurus (Rafinesque, 1814) (Rodentia, Muridae). Experientia 34: 325-328.

Raman, R. and Sharma, T. 1974. DNA replication, G- and C-bands and meiotic behaviour of supernumerary chromosomes of Rattus rattus (Linn.). Chromosoma (Berl.) 45: 111119.

Schwarzacher, H. G., Mikelsaar, A. V. and Schnedl, W. 1978. The nature of the Ag-staining of 
nucleolus organizer regions. Cytogen. Cell Genet. 20: 24-39.

Seabright, M. 1971. A rapid banding technique for human chromosomes. Lancet 11: 971-972.

Sumner, A. 1972. A simple technique for demonstrating centromeric heterochromatin. Exp. Cell Res. 75: 304-306.

Vistorin, G., Gamperl, R. and Rosenkranz, W. 1978. Comparison of the karyotypes of Rattus rattus flavipectus (Rodentia) and European rats. Zool. Anz., Jeana 201: 314-322.

Yosida, T. H. 1973. Evolution of caryotypes and differentiation in 13 Rattus species. Chromosoma (Berl.) 40: 285-297.

- 1975. Diminution of heterochromatic C-bands in relation to differentiation of Rattus species. Proc. Japan Acad. 51 : 659-663.

- 1977a. Frequencies of chromosome polymorphism in pairs no. 1,9 and 13 in three geographical variants of black rats, Rattus rattus. Chromosoma (Berl.) 60:391-398.

- 1977b. Supernumerary chromosomes in the black rat (Rattus rattus) and their distribution in three geographical variants. Cytogenet. Cell Genet. 18: 149-159.

- 1978. A preliminary note on silver-stained nucleolar organizor regions in the black and Norway rats. Proc. Jap. Acad. Ser. B 54: 353-358.

-1979a. Genetic aspect of polymorphic C-bands in the chromosomes of the black rat (Rattus rattus tanezumi) in Japan. Cytologia 44: 265-274.

- 1979b. Comparative study on nucleolus organizer regions (NORs) in 7 Rattus species with special emphasis on the organizer differentiation and species evolution. Proc. Jap. Acad.

Ser. B 55 : $481-486$.

- and Sagai, T. 1972. Banding pattern analysis of polymorphic karyotypes in the black rat by a new differential staining technique. Chromosoma (Berl.) 37: 387-394.

- and - 1973. Similarity of Giemsa banding patterns of chromosomes in several species of the genus Rattus. Chromosoma (Berl.) 41 : 93-101.

- and - 1975. Variation of C-bands in the chromosomes of several subspecies of Rattus rattus. Chromosoma (Berl.) 50: 283-300.

-and Parida, B. B. 1980 . Karyotype evolution, species differentiation and environmental mutagen. Proc. Japan Acad. Ser. B 56: 79-84.

-, Tsuchiya, K. and Moriwaki, K. 1971. Karyotypic differences of black rats, Rattus rattus, collected in various localities of East and Southwest Asia and Oceania. Chromosoma (Berl.) 33: 252-267.

-, Kato, H., Tsuchiya, K., Sagai, T. and Moriwaki, K. 1974. Cytogenetical surgery of black rats, Rattus rattus, in Southwest and Central Asisa, with special regard to the evolutional relationship between three geographical types. Chromosoma (Berl.) 45: 99-109.

,,--- Moriwaki, K., Ochiai, Y. and Monty, J. 1979. Black rats from Mauritus with a new karyotype involving the Robertsonian fission. Proc. Japan Acad. Ser. B 55: 120-125. 